\title{
Survey in Existing Non-Local Means Algorithm for Noise Reduction
}

\author{
Arti Singh \\ Research Scholar \\ Computer Science \\ BBAU University, \\ Lucknow Uttar Pradesh India
}

\author{
Ram Singar Verma \\ Assistant Professor \\ Computer Science \\ BBAU University, \\ Lucknow Uttar Pradesh India
}

\begin{abstract}
This paper introduced the concept of noise reduction for recovering the original image. Digital images are a very important role in daily life like satellite television, computer etc. Sometimes digital images are faces problem called noise. For this problem, we study non-local means algorithm. In this algorithm uses a self-similarity concept, called "non-local means algorithm". Image accommodates noise like Gaussian noise, salt \& pepper noise, speckle noise, film grain etc. In this paper, only survey on the existing non-local means algorithm for noise reduction which is taken from many devices like camera or other digital gadgets.
\end{abstract}

\section{Keywords}

Gaussian noise, speckle noise, salt \& pepper noise, Non-local mean algorithm, Noise reduction

\section{INTRODUCTION}

Image processing always allows a use of more complex algorithm. Digital image processing is complicated programming. Images which is captured by digital cameras, scanners etc. Noise in image easily entered; unfortunately, images are degraded by noise during the transmission process. So noise reduction of the image has been must important studied problem in noise reduction. Recovering original image by removing noise from the image is challenging problem in digital image processing. An image is surely corrupted by noise during capturing, coding and transmission. During capturing image from camera's or other systems are influenced by many other factors like environmental conditions. Such as light level or sensors temperature which produces noise in an image. During transmission of the image, images are corrupted due to the disturbance in the channel used for communication \& transmission. The main objective of noise reduction is "removing noise from image".

Many algorithms for noise reduction make two premises for a noisy image. These premises are blurring and loss of details;

The first premise is: if noise is present in the image, i.e. white noise that means noise accommodates all frequencies high \& low because of the higher frequency, called oscillatory other terms "non-smooth".

Second premise is: True images (without noise in the image) are smooth or piecewise smooth means that patches of a true image consist of only a few frequencies.

In this paper, we intentional about non-local means algorithm for noise reduction which take the mean value of a group of pixels enclosing a target pixel in the image to smooth. In nonlocal mean algorithm takes a mean of all pixels the image weighted by how similar these pixels are to a target pixel. These results provide much greater post-filtering clarity and less loss of details. Noise degraded image quality. This algorithm mainly used for remove Gaussian, speckle noise. Gaussian noise is used as additive white noise. Each pixel in the noisy image is the sum of pixels value and random Gaussian distributed noise value. Speckle noise is granular noise and degraded image quality. It is a method as spatial correlated multiplicative noise presented in all steps of image acquisition.

\section{RELATED WORK}

[1-2] "Non local algorithm for noise reduction in image proposed by Antoni Buades, B. Coll \& J. Michel morel. In this algorithm, propose a new concept for removing noise, evaluate and compare the performance of digital noise reduction of image. [13] B. Goossens, H. Luong ,A. pizurica and $\mathrm{W}$. Philips proposed improved non-local noise reduction algorithm for image. In this algorithm presented some improvement with original non-local algorithm and add extension colored noise for noise reduction , for Gaussian noise show PSNR result, proposed algorithm is better than BM-3D method and visual quality is better and also colored noise some improvement in proposed algorithm performance to recent wavelet based techniques. [5] T. Tasdizen proposed for Principal neighborhood dictionaries non-local mean noise reduction for image .Presented in depth analysis of non-local means noise reduction algorithm which is using principal component analysis (PCA) for higher accuracy with reducing Computational load. The accuracy of NLM is image neighborhood \& also accuracy of PND is examined by search windows sizes. In this algorithm discussing comparison of PND; NLM \& other image neighborhood or PCA based noise reduction algorithm. [6] R. vignesh ,B. Tae oh and C.C JAY Kuo presented new algorithm in terms of speed fast non-local means with PET(probabilistic early termination) .In this algorithm specific amount of computation in non-local means scheme is based on contortion calculation between pixel neighborhoods. PET algorithms adopts probability model for goal early termination algorithm. Comparison with others fast non-local means for providing demonstrate the effectiveness of algorithm.[12] J. Wang,Y. Guo ,Y. Ying, Yanli Liv, Q. Peng proposed new algorithm fast non-local algorithm for noise reduction in image, presented new algorithm for reducing the computational cost . Calculate similarity of neighborhood windows is proposed .This algorithm result provide fifty times faster than original non-local algorithm ,both based on theoretically \& experimentally in terms on mean squared error and percentage image quality.

\section{TYPES OF NOISE}

Removing noise may be possible with histogram or probability density function. The noise is calculated by:

$\mathbf{W}(\mathbf{x}, \mathbf{y})=\mathbf{U}(\mathbf{x}, \mathbf{y})+\mathbf{V}(\mathbf{x}, \mathbf{y})$ 
Where, $\mathrm{P}(\mathrm{x}, \mathrm{y})$ is Original image pixel value, $\mathrm{Q}(\mathrm{x}, \mathrm{y})$ is noise and $\mathrm{R}(\mathrm{x}, \mathrm{y})$ is resulting in noise image.

\subsection{Gaussian noise}

Gaussian noise is statistical noise \& also known as "Gaussian distribution". The values that the noise can take on are Gaussian distributed statistical noise having a probability density function equal to the normal distribution.

$$
\mathbf{G}(\mathbf{x})=\frac{1}{\sigma \sqrt{2 \pi}} e^{-(x-\mu)^{2} / 2 \sigma^{2}}
$$

Where $\mathrm{x}$ is gray level, $\mu$ is mean value and is standard derivation.

\subsection{Speckle noise}

Speckle noise is also known as multiplicative noise.Multiplicative noise is a granular noise that degrades the quality of the active radar, etc. This type of noise can't observe by human eyes.This noise follows a gama distribution:

$$
\mathbf{G}(\mathbf{x})=\frac{x^{\alpha-1}}{(\alpha-1) ! a^{\alpha}} e^{x / a}
$$

Where $a^{2} \alpha$ is variance and $\mathrm{x}$ is gray level

\section{NON-LOCAL MEANS BASED ALGORITHM FOR NOISE REDUCTION}

\subsection{Initial non-local means algorithm:}

Antoni Buades etc is firstly proposed non-local means concept for noise reduction.

Given a noisy image $p=\{p(i) / i \in I$, the estimated value $N L[p](i)$, for a pixel $i$ is computed as a weighted average of all the pixels in the image,

$$
N L[p](i)=\sum_{j \in I} w(i, j) p(j),
$$

Where, in image weights $\{w(i, j)\}_{j}$ depends on similarity between pixels $i$ and $\mathrm{j} \&$ must satisfy condition

$$
0 \leq w(i, j) \leq 1 \& \& \quad \sum_{j} w(i, j)=1
$$

Between two pixels $i \& j$ finds similarity depends on the similarity of intensity gray level vectors $p\left(N_{i}\right) \& p\left(N_{j}\right)$, where $N_{k}$ denotes a square neighborhood of fixed size \& centered at pixels.

Measuring similarity between pixels as a decreasing function of the weighted Euclidean distance $\| p\left(N_{i}\right)-$ $\left.p\left(N_{j}\right)\right|_{2, a} ^{2}$, where a>0 is the standard deviation of the Gaussian kernel. Euclidean distance to noisy neighborhoods raises the following equality

$$
\begin{gathered}
E\left\|p\left(N_{i}\right)-p\left(N_{j}\right)\right\|_{2, a}^{2}=\left\|q\left(N_{i}\right)-q\left(N_{j}\right)\right\|_{2, a}^{2}+2 \sigma^{2} \\
\ldots \text { (1) }
\end{gathered}
$$

Equation(1) shows that robustness of algorithm, so Euclidean distance conserves the order of similarity $b / w$ pixels.

The above mentioned weights are defined as:

$$
w(i, j)=\frac{1}{Z(i)} e^{-\frac{\left\|p\left(N_{i}\right)-p\left(N_{j}\right)\right\|_{2, a}^{2}}{h^{2}}},
$$

Where $(i)$ : normalizing constant

$$
Z(i)=\sum_{j} e^{-\frac{\left\|p\left(N_{i}\right)-p\left(N_{j}\right)\right\|_{2, a}^{2}}{h^{2}}}
$$

Where, parameter $\mathrm{h}$ :degree of filtering.

The NL-means algorithm not only compared the gray level in a single point but the geometrical configuration in a whole neighborhood. This information allows robust comparison than neighborhood filters.

Drawbacks in NL-means algorithm: Some drawbacks exist in the Original NL-means algorithm. These points defined below:

- In NL mean algorithm violated pixels are retrieved from the weighted average of the similar pixels.

\subsection{Improved Fast Non-local means algorithm}

This algorithm is proposed by Jin-Wang, Yanwen Guo, etc. In this algorithm reducing the computational cost \& this cost for calculating the similarity of neighborhood windows.

In this algorithm, introducing approximate measurement about the similarity of neighborhoods windows and use efficient summed square image scheme and Fast Fourier transform. This algorithm providing fifty times better result than original non-local means algorithm.

For compare two neighborhoods, we take Euclidean distance:

$S(i, j)=\left\|N_{i}-N_{j}\right\|^{2}=\sum_{l=0}^{M-1} \sum_{m=0}^{M-1}\left[I_{i}(l, m)-I_{j}(l, m)\right]^{2}$

Where $\left\{\begin{array}{l}\boldsymbol{I}_{\boldsymbol{i}}(\boldsymbol{l}, \boldsymbol{m}) \\ \boldsymbol{I}_{\boldsymbol{j}}(\boldsymbol{l}, \boldsymbol{m})\end{array}\right\}$ represents pixels in $\boldsymbol{N}_{\boldsymbol{i}} \& \boldsymbol{N}_{\boldsymbol{j}} \cdot \boldsymbol{I}_{\boldsymbol{j}}(\boldsymbol{l}, \boldsymbol{m})$ Can be represented in global coordinates on mirrored image as:

$$
I_{j}\left(l-a_{j}^{\prime}, m-b_{j}^{\prime}\right),
$$

Where, $\quad a_{j}^{\prime}=3 M / 2+a_{j}$

$$
b_{j}=3 M / 2+b_{j}
$$

So equation is,

$$
\begin{gathered}
S(i, j)=\sum_{l=0}^{M-1} \sum_{m=0}^{M-1}\left[I_{i}(l, m)-I_{j}\left(l-a_{j}, m-b_{j}^{\prime}\right)\right]^{2} \\
=N_{i}^{2}+N_{j}^{2}-N_{i} * N_{j}
\end{gathered}
$$

$N_{i} * N_{j}$ Provides multiplication under the fast Fourier transform. $N_{i}^{2} \& N_{j}^{2}$ Can be fast calculated as well using summed square image (SSI).

\subsubsection{Summed square image (SSI)}

In this algorithm use summed square image. The SSI follows integral image which used for face detection techniques. In the image, every pixel integral image manages the summed value of all pixels in the upper left part of the original image. Here we expand it to SSI.As an integral image, for every pixel $(a, b)$ SSI stored sum for squared values of upper left pixels. 


$$
\operatorname{SSI}\left(a_{0}-b_{0}\right)=\sum_{a \leq a_{0}, b \leq b_{0}} I^{2}(a, b)
$$

Calculated efficiency:-

For $a_{0}=0, b_{0}=0$

$$
\operatorname{SSI}(0,0)=I^{2}(0,0)
$$

For $a_{0}>0, b_{0}=0$

$$
\operatorname{SSI}\left(a_{0}, 0\right)=\operatorname{SSI}\left(a_{0}-1,0\right)+I^{2}\left(a_{0}, 0\right)
$$

For $a_{0}=0, b_{0}>0$

$$
\operatorname{SSI}\left(0, b_{0}\right)=\operatorname{SSI}\left(0, b_{0}-1\right)+I^{2}\left(0, b_{0}\right)
$$

For $a_{0}>0, b_{0}>0$

$$
\begin{gathered}
S S I\left(a_{0}, b_{0}\right)=S S I\left(a_{0}-1, b_{0}\right)+S S I\left(a_{0}, b_{0}-1\right)- \\
S S I\left(a_{0}-1, b_{0}-1\right)+I^{2}\left(a_{0}, b_{0}\right)
\end{gathered}
$$

In this above algorithm in the original image, every pixel proposed only once. So computed complexity is $O\left(n^{2}\right)$, where $n^{2}$ is the size of the image.

\subsection{Bayesian-based Non-local means scheme}

This approach is proposed by Charles, etc .we used Bayesian approach for a non-local mean filter. This approach focused on how to improve the performance of this filter by adaptive local dictionaries and new statistical distance measure for compare patches. This approach includes:

- Partition the $\boldsymbol{\Omega}$ image into overlapped blocks $A_{p r}$,

Size $X=(2 \propto+1)^{g}$

Where, g: dimensionality of image and $\boldsymbol{\Omega}=\mathbf{Y}_{\mathbf{r}} \mathbf{A}_{\mathbf{p r}}$

- Block $A_{p r}$ restored based on non-local means algorithm:

$$
\begin{gathered}
\boldsymbol{N L}(\boldsymbol{a})\left(A_{p r}\right)=\sum_{A_{q} \in Z_{p r}} \boldsymbol{w}\left(S_{p r}, S_{q}\right) \boldsymbol{a}\left(A_{q}\right) \\
\boldsymbol{w}\left(\boldsymbol{S}_{p r}, S_{q}\right)=\frac{1}{K_{p r}} e^{-\frac{\left\|a\left(A_{p r}\right)-a\left(A_{q}\right)\right\|_{2}^{2}}{h^{2}}}
\end{gathered}
$$

Where $a\left(A_{p}\right)=\left(a^{(1)}\left(A_{p}\right)----a^{(X)}\left(A_{p}\right)\right)^{T}$ is image patch.

- Pixel values are restoring based on to restored intensities of blocks which belong to pixel so restored intensity of pixels $S_{p}$ is

$$
N L(a)\left(S_{p}\right)=\frac{1}{\left|B_{p}\right|} \sum_{p \in B_{p}} B_{p}
$$

Bayesian interpretation of NL-means algorithm block-wise non-local means filter is:

$$
=\frac{N L(a)\left(A_{p r}\right)}{\frac{1}{\left|Z_{p r}\right|} \sum_{q=1}^{\left|Z_{p r}\right|} p\left(a\left(A_{p r}\right) \mid a\left(A_{q}\right)\right) p\left(a\left(A_{q}\right)\right)\left(a\left(A_{q}\right)\right.}
$$

Where

$$
\begin{aligned}
& p\left(a\left(A_{p r}\right) \mid a\left(A_{q}\right)\right) \text { and } p\left(a\left(A_{q}\right)\right)::: \\
& \text { distribution of } \left.a\left(A_{p r}\right) \mid a\left(A_{q}\right)\right) \text { and prior distribution of patches }
\end{aligned}
$$

The algorithm performed on synthetic data with a different type of noise levels and speckle simulation. Results show that classical implementation of NL mean algorithm as well as speckle reducing anisotropic diffusion and squeeze box filters.

\subsection{Probabilistic early Termination based Non-local mean algorithm}

PET is proposed by Ramanathan etc for speeding up.PET support a probability model to obtain the early termination. If expected distortion value is high to be of significance in weighted averaging, So it is the special case for distortion computation can be stopped/terminated and the corresponding contributing pixel can be rejected.

This scheme used for eliminating dissimilar blocks without full computation of distortion. If the probability is greater than fixed value $P_{x}$. So finding the probability of distortion value and stop distortion computation.

- Resolution of summation order: Determine a $\sum$. order for the point in A.It is used radial scanning order where starting from inner-most regions and moving towards outer regions.

Mathematically, decomposing set A,

$$
\mathrm{A}=\mathrm{A} \cup \ldots \ldots \ldots \cup A_{n} \cup A_{g},
$$

Where, $A_{n}=\left\{a \in A \mid(n-1)^{2} \leq\|a\|^{2}<n^{2}\right\}, 1 \leq n \leq g$ So, distortion sum for every $A_{n}$ is :

$$
d_{n}(i, j)=\sum_{a \in A_{n}} G_{m}(a)(Y(i-a)-Y(j-a))^{2}
$$

And partial sum from $A_{1}$ to $A_{n}$ :

$$
D_{n}(i, j)=d_{1}(i, j)+\cdots \ldots+d_{n}(i, j)
$$

- Statistical characterization of patch differences: The distortion can be interpreted as the energy of weighted batch-difference.

$$
Y_{i j}(a)=\sqrt{a_{m}(a)}(Y(i-a)-Y(j-a))
$$

Where, $a \in A$.It is used for describing SAD distribution over the different block.Here the value of $D_{g}(i, j)$ is used for select blocks in weighted averaging.we try to find total sum $D_{g}$ from partial sum $D_{n}$. The $\chi^{2}$ distribution is distance b/w two image patches for calculated adaptive weights i.e.

We assume, $\boldsymbol{Y}_{\boldsymbol{i}, \boldsymbol{j}} \sim \boldsymbol{G}\left(\boldsymbol{O}_{\boldsymbol{i j}}, \boldsymbol{\sigma}_{\boldsymbol{i j}}^{2}\right)$, the sum of $Y_{i j}^{2}$ follow $\chi^{2}$ distribution.

The above idea used to reject dissimilar patch at earlier termination scheme as: $\quad \boldsymbol{D}_{\boldsymbol{n}}(\boldsymbol{i}, \boldsymbol{j})>\delta$

In contrast, PET estimates probability distribution of distortion remaining region:

$\boldsymbol{R}_{\boldsymbol{n}}(\boldsymbol{i}, \boldsymbol{j})=\boldsymbol{d}_{\boldsymbol{n}+\mathbf{1}}(\boldsymbol{i}, \boldsymbol{j})+\cdots+\boldsymbol{d}_{\boldsymbol{g}}(\boldsymbol{i}, \boldsymbol{j})$, Rejected patches when $\boldsymbol{P}\left(\boldsymbol{R}_{\boldsymbol{n}}(\boldsymbol{i}, \boldsymbol{j})+\boldsymbol{D}_{\boldsymbol{n}}(\boldsymbol{i}, \boldsymbol{j})>\tau \mid \boldsymbol{D}_{\boldsymbol{n}}(\boldsymbol{i}, \boldsymbol{j})\right)>\boldsymbol{P}_{T}$

- Resolution of mean and variance: Find mean $O_{i j}$ and variance $\boldsymbol{\sigma}_{\boldsymbol{i} \boldsymbol{j}}^{2}$ for Gaussian random variable $\boldsymbol{Y}_{\boldsymbol{i} \boldsymbol{j}}$

The patch difference mean: $\boldsymbol{O}_{\boldsymbol{i j}}=\boldsymbol{O}_{\boldsymbol{i}}-\boldsymbol{O}_{\boldsymbol{j}}$ where

$$
\begin{aligned}
& \boldsymbol{m}_{\boldsymbol{i}}=\frac{\sum_{a \epsilon A} \sqrt{\boldsymbol{G}_{\boldsymbol{m}}(\boldsymbol{a})} \boldsymbol{Y}(\boldsymbol{i}-\boldsymbol{j})}{|\boldsymbol{A}|}, \\
& |.| \text { is cardinality of set }
\end{aligned}
$$

Variance: $\sigma_{i j}^{2}=E\left(Y_{i j}^{2}\right)-\left(E\left(Y_{i j}\right)\right)^{2}$

Where (.) : Expectation of variable 
$E\left(Y_{i j}^{2}\right):$ Average of $Y_{i j}^{2}(a), a \in A_{1} \cup \ldots . . \cup A_{n}$

So results finally show that PSNR quality improved and visual perception enhanced.

\section{CONCLUSION}

In this algorithm "A non local mean algorithm for image denoising" [1].The NL-means algorithm not only compared the gray level in a single point but the geometrical configuration in a whole neighborhood. This information allows robust comparison than neighborhood filters.

In this algorithm "FAST NON-LOCAL ALGORITHM FOR IMAGE DENOISING"[7].In this algorithm improving existing original algorithm "A non-local mean for image denoising"[].Improve non-local mean in terms of speed calculation. It is based on SUMMED SQUARE IMAGE and FAST FOURIER TRANSFORM. This algorithm accelerated fifty times of original algorithm for noise reduction.

Probabilistic Early Termination used for fast NON LOCAL MEAN computation If partial sum of distortion term increase with pre-defined threshold. So terminate the computation of distortion. In this algorithm, there are two main points for considering : complexity reduction, improve PSNR quality.

Bayesian Non local mean filter introduced how improve performance of filter for noise reduction .In this algorithm, we find better parameters for filter size of adaptive dictionary and noise variance are parameter for filter and computed from input image .most important thing patch size must larger. This Bayesian algorithm has used for removing dependent noise in image.

\section{FUTURE WORK}

Basically, in this paper only showing review of existing algorithm but in future Matlab code upgraded in VHDL code and implementation for image transformation and analysis in FPGA kit to develop ASIC which is using for special work of noise reduction, which person don't know about any algorithm of noise reduction in image are also capable of doing it.

\section{REFERENCES}

[1] Antoni Buades, Jean Michel Morel, "A non-local algorithm for image denoising", Proc. Int. Conf. Computer Vis. Pattern Recognition, 2005, pp. 60-65
[2] Antoni Buades, B. Coll, and J. Morel. Neighborhood filters and pde's. Technical Report 2005-04, CMLA, 2005.

[3] T. Tasdizen, "Principal Neighborhood Dictionaries for Non-local Means Image Denoising”, IEEE TRANSACTIONS ON IMAGE PROCESSING, VOL. XX, NO. X, JANUARY 2009.

[4] Ramanathan Vigneshy, Byung Tae Oh_ and C.-C.Jay Kuo, "Fast Non-Local Means Computation with Probabilistic Early Termination", IEEE Signal Processing Letters

[5] A A Kervrann, J. Boulanger, P. Coupe, "Bayesian nonlocal means filter, image redundancy and adaptive dictionaries for noise removal", International conference on scale space and variational methods in computer vision, 2007

[6] R. Lai, Xuan-xuan Dou, "Improved non-local means filtering algorithm for image denoising", International Congress on Image and Signal Processing (CISP) 2010.

[7] Y. Yue, M. M. Croitoru, A. Bidani, Joseph B. Z wischenberger and John W. Clark, " Nonlinear multiscale diffusion for speckle suppression and edge enhancement in ultrasound images", IEEE Transactions on Medical Imaging, vol.25, 2006, pp. 297-311.

[8] Liu YL, Wang J, Chen X etc al. " A robust and fast nonlocal means algorithm for image denoising”. JOURNAL OF COMPUTER SCIENCE AND TECHNOLOGY 23(2): 270 \{279 Mar. 2008.

[9] J. Portilla, V. Strela, M. Wainwright, \& E. Simoncelli, "Image denoising using scale mixtures of Gaussians in the wavelet domain ," IEEE Transaction on image processing 12(11), pp. 1338-1351,2003.

[10] Huang J, Mumford D. Statistics of natural images and models. ICCV. 1999:541-547.

[11] J. Wang,Y. Guo ,Y. Ying , Yanli Liv , Q. Peng Mahmoudi M, Sapiro G. "Fast NON-LOCAL ALGORITHM FOR IMAGE DENOISING" IEEE Signal Processing Letters. 2005;12(12):839-842.

[12] Dauwe, B. Goossens, H. Luong and W. Philips, " a Fast Non-Local Image Denoising Algorithm," SPIE-IS\&T Vol. 6812 